\title{
FURTHER DETECTIONS OF OH MASERS IN CARBON STARS WITH SILICATE FEATURES
}

\author{
M. Szymczak \\ Toruń Centre for Astronomy, Nicolaus Copernicus University, Toruń, Poland \\ R. Szczerba \\ Nicolaus Copernicus Astronomical Center, PAS, Toruń, Poland
}

P.S. Chen

Yunnan Astronomical Observatory, CAS, Kunming, P.R. China

\begin{abstract}
A sample of J-type carbon stars was searched for $\mathrm{OH}$ maser emission. The new detection of three $\mathrm{OH}$ lines towards two silicate carbon stars is reported. In V778 Cyg, previously known as the main-lines (1665 and $1667 \mathrm{MHz}$ ) maser source, the satellite $1612 \mathrm{MHz}$ emission was discovered while in NSV 2814 the main $\mathrm{OH}$ lines were detected. The presence of $\mathrm{OH}$ maser lines confirms the former suggestion that oxygen-rich material is located in the vicinity $\left(\approx 10^{15-16} \mathrm{~cm}\right)$ of silicate carbon stars.
\end{abstract}

\section{INTRODUCTION}

The silicate emission features at about 10 and $18 \mu \mathrm{m}$ are characteristic for oxygen-rich dust envelopes. However, these features were also discovered in some optically classified carbon stars (Little-Marenin 1986; Willems \& de Jong 1986), named later silicate carbon stars. Furthermore, many silicate carbon stars are recognized as ${ }^{13} \mathrm{C}$-rich (J-type) carbon stars (Lambert et al. 1990; Lloyd Evans 1990 and references therein). The most recent compilation of 22 known and suspected silicate carbon stars is presented by Chen et al. (1999). Note also the discovery of the first extragalactic silicate carbon star (IRAS 04496-6958) in the Large Magellanic Cloud (Trams et al.1999). The detection of silicate emission from some (silicate) carbon stars suggests that their relatively close surroundings contain oxygen-based dust, in spite of their photospheric 
chemical composition which shows $\mathrm{C} / \mathrm{O}>1$. An additional argument for the existence of oxygen-rich material in the vicinity of these stars comes from the detection of water and hydroxyl maser lines towards some of them (see Little-Marenin et al. 1994 and Engels 1994 and references therein). To date $\mathrm{H}_{2} \mathrm{O}$ masers were found in four (J-type) silicate carbon stars and only one of them (V778 Cyg) exibits $\mathrm{OH}$ maser emission in the main-lines. In addition $1612 \mathrm{MHz}$ maser emission was detected in FJF 270 (te Lintel Hekkert 1991). Here we report the results of a high sensitivity search for $\mathrm{OH}$ emission towards $14 \mathrm{~J}$-type carbon stars including 7 known silicate carbon stars.

\section{OBSERVATIONS}

The observations were performed with the Nançay radio telescope on April $6-13$, 1999. The HPBW was $3.5^{\prime}$ in right ascension by $18^{\prime}$ in declination. A dual channel receiver was used. The system temperature was about $50 \mathrm{~K}$. The ratio of flux density to antenna temperature was $1.1 \mathrm{Jy} \mathrm{K}^{-1}$ at $0^{\circ}$ declination. The 1024 -channel autocorrelation spectrometer was split into 4 banks, each covering a bandwidth of $0.2 \mathrm{MHz}$, for the three $\mathrm{OH}$ lines. The $1612 \mathrm{MHz}$ satellite line was observed in both circular polarizations while the 1665 and $1667 \mathrm{MHz}$ main-lines were observed in left and right circular polarization, respectively. The spectra were taken in frequency switching mode with channel spacings of 0.28 and $0.29 \mathrm{~km} \mathrm{~s}^{-1}$ at the main-lines and the satellite line, respectively. Upper flux density limit for the non-detections was 80 mJy $(3 \sigma)$. W 12 was observed to provide the flux density calibration which was accurate to about $10 \%$.

\section{RESULTS AND DISCUSSION}

Our program stars are listed in Table 1 with the designation from the General Catalogue of Cool Galactic Carbon Stars (CCGCS, Stephenson 1989) given in the first column. Known silicate carbon stars are marked in boldface. Previously detected $\mathrm{H}_{2} \mathrm{O}$ and/or $\mathrm{OH}$ maser lines are marked by a ' + ' sign while our new detections are depicted by a ' ++ ' sign. $\mathrm{OH}$ masers were found in NSV 2814 (main-lines) and in V778 Cyg (satellite line). Note that the $1612 \mathrm{MHz}$ maser line towards V778 Cyg has not been detected either by Barnbaum et al. (1991) nor by Little-Marenin et al. (1994).

The spectra are shown in Fig. 1 where, for the sake of comparison, re-observed $\mathrm{OH}$ main-lines from V778 Cyg are presented. In V778 Cyg the $1667 \mathrm{MHz}$ feature at $-16.0 \mathrm{~km} \mathrm{~s}^{-1}$ has a peak flux density of $0.30 \mathrm{Jy}$, which is a factor of 1.5 higher than the $1665 \mathrm{MHz}$ peak of $0.19 \mathrm{Jy}$ at 
Table 1 List of observed stars.

\begin{tabular}{llcccc}
\hline CCGCS & other name & OH 1612 & OH 1665 & OH 1667 & $\mathrm{H}_{2} \mathrm{O}$ \\
\hline $\mathbf{1 1 5 8}$ & NSV 2814 & - & ++ & ++ & + \\
$\mathbf{1 6 5 3}$ & BM Gem & - & - & - & - \\
1682 & & - & - & - & + \\
1698 & & - & - & - & + \\
1921 & & - & - & - & - \\
2028 & & - & - & - & - \\
2156 & MT Hya & - & - & - & - \\
3066 & HD 100764 & - & - & - & - \\
$\mathbf{3 9 3 5}$ & FJF 270 & + & - & - & - \\
$\mathbf{4 2 2 2}$ & NC 83 & - & - & - & - \\
$\mathbf{4 9 2 3}$ & V778 Cyg & ++ & + & + & + \\
$\mathbf{5 5 2 6}$ & MQ Cyg & - & - & - & - \\
5548 & V1415 Cyg & - & - & - & + \\
$\mathbf{5 8 4 8}$ & EU And & - & - & - & + \\
\hline
\end{tabular}
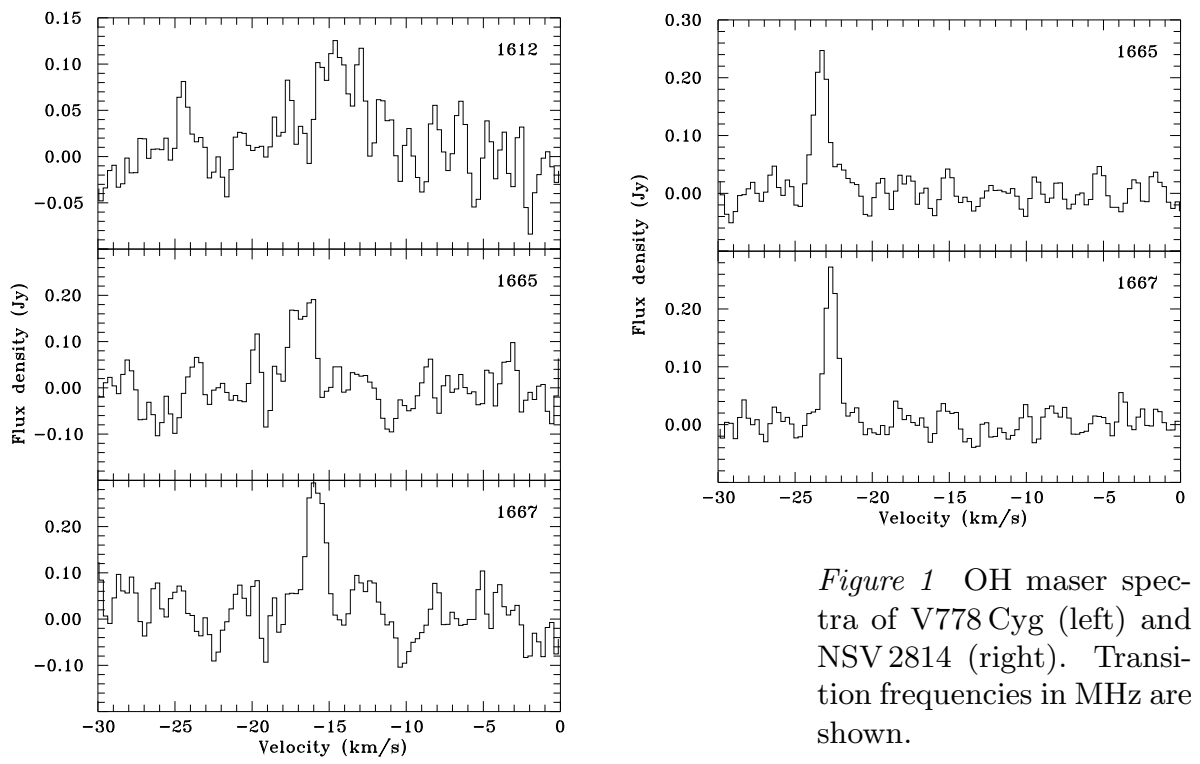

Figure 1 OH maser spectra of V778 Cyg (left) and NSV 2814 (right). Transition frequencies in $\mathrm{MHz}$ are shown.

$-16.9 \mathrm{~km} \mathrm{~s}^{-1}$. Within the measurement errors the intensities and radial velocities of these lines are the same as observed 12 years ago (Little-Marenin et al. 1988). The $1612 \mathrm{MHz}$ feature of $0.12 \mathrm{Jy}$ peaks at $-14.4 \mathrm{~km} \mathrm{~s}^{-1}$. This indicates that the $1612 \mathrm{MHz}$ emission is slightly red- 
shifted relatively to the $\mathrm{OH}$ main-lines but coincides with a $-14 \mathrm{~km} \mathrm{~s}^{-1}$ component of the $\mathrm{H}_{2} \mathrm{O}$ maser (Engels \& Leinert 1994).

In NSV 2814 the peak intensities of the 1665 and $1667 \mathrm{MHz}$ are 0.24 and $0.27 \mathrm{Jy}$, respectively. The velocities of $\mathrm{OH}$ features at -23.5 and $-22.8 \mathrm{~km} \mathrm{~s}^{-1}$ are very different from the $\mathrm{H}_{2} \mathrm{O}$ maser velocity of $-13 \mathrm{~km} \mathrm{~s}^{-1}$ detected about 7 years ago (Engels 1994). Although temporal variations may affect the maser profiles, the difference in velocities of the $\mathrm{OH}$ and $\mathrm{H}_{2} \mathrm{O}$ maser features of about $10 \mathrm{~km} \mathrm{~s}^{-1}$ is quite high for an AGB object. This may suggest that each maser is associated with a different object. Little-Marenin et al. (1994) noted that CCGCS 1158 may be incorrectly associated with the IRAS source. Furthermore, the intensity ratio of $1667 / 1665 \mathrm{MHz}$ transitions of about 1.1 suggests optically thin LTE conditions, so that the masers may arise in the intervening interstellar medium. The case of NSV 2814 needs further careful studies.

In spite of almost 15 years of debate, the formation of silicate carbon stars still remains controversial. A stability of the silicate feature (Yamamura et al. 2000) and $\mathrm{OH}$ main-lines (this work) in V778 Cyg makes the scenario of fast transition from M-type AGB stars into carbon stars difficult to reconcile with the lifetime of silicate emission from an expanding detached shell (see e.g. Yamamura et al.2000). A plausible scenario may be some kind of long-lived reservoirs of oxygen-rich material in a binary system (e.g. Lloyd Evans 1990 and Jura \& Kahane 1999) with (possibly) a main sequence secondary (e.g. Lambert et al. 1990 and Yamamura et al. 2000). Silicate carbon stars seem to be related to the normal J-type carbon stars in the sense that both groups show $\mathrm{H}_{2} \mathrm{O}$ maser emission (Engels 1994) and that the mechanism responsible for their low ${ }^{12} \mathrm{C} /{ }^{13} \mathrm{C}$ ratios might be the same (Ohnaka \& Tsuji 1999).

\section{Acknowledgments}

The authors acknowledge support from grant No. 2.P03D.002.13 of the Polish State Committee for Scientific Research.

\section{References}

Barnbaum C., Morris M., Likkel L, et al., 1991, A\&A 251, 79

Chen P.S., Wang X.H., Wang F., 1999, Chin. Astron. Astrophys. 23, 371

Engels D., 1994, A\&A 285, 497

Engels D., Leinert Ch., 1994, A\&A 282, 858

Jura M., Kahane C., 1999, ApJ 521, 302

Lambert D.L., Hinkle K.H., Smith V.V., 1990, AJ 99, 1612

Little-Marenin I.R., 1986, ApJ 307, L15

Little-Marenin I.R., Benson P.J., Dickinson D.F., 1988, ApJ 330, 828 
Little-Marenin I.R., Sahai R., Wannier P.G., et al., 1994, A\&A 281, 451 Lloyd Evans T., 1990, MNRAS 243, 336

Ohnaka K., Tsuji T., 1999, A\&A 345, 233

Stephenson C.B., 1989, Pub. Warner and Swasey Obs., Vol. 3, No.2 (CCGCS)

te Lintel Hekkert P., Caswell J.L., Habing H.J., et al., 1991, A\&AS 90, 327

Trams N.R., van Loon J.Th., Zijlstra A.A., et al., 1999, A\&A 344, L17

Willems F.J., de Jong T., 1986, ApJ 309, L39

Yamamura I., Dominik C., de Jong T., et al., 2000, A\&A in press 